\title{
REFLECTION
}

\section{The Return Visit}

\author{
Mitchell Charap, MD
}

Department of Medicine, New York University School Medicine, 550 First Avenue, New York, NY 10016, USA.

DOI: $10.1007 / \mathrm{s} 11606-007-0146-\mathrm{Z}$

(C) 2007 Society of General Internal Medicine 2007;22:553

I was surprised to hear that a patient of mine, Mr. Cardosa, had died. I had not even realized that he was hospitalized. When his wife called, she apologized for not letting me know. His gastric cancer had spread, and after watching him deteriorate rapidly, Mrs. Cardosa recalled their prior discussions and opted against further treatment.

She was wracked with guilt. She had felt very isolated in the hospital and believed that the oncologist disagreed with her decision to stop the chemotherapy. I told her that I agreed with her, but I felt miserable about my role, or more accurately, the absence of my role in his care.

I tried to reconstruct what had happened 10 months earlier after I told Mr. Cardosa his diagnosis. I recalled reassuring him and arranging for a consultation with a GI oncologist. It was my usual practice to schedule all patients for a return visit. But this time I did not. I reasoned that other than the cancer, he had no active medical problems; his next several months would be occupied with clinical issues related to his cancer. Looking back, I believe that there were other reasons. First, I was not comfortable in being a bystander in clinical decisions, and second, on some level, I was relieved that someone else would now be caring for him.

A few months later, I received a detailed consult from the oncologist which noted his uneventful surgery and outlined the chemotherapy protocol. I did not hear from the Cardosas until 6 months later, when Mrs. Cardosa called to informed me that her husband was losing weight and unable to eat. Mr. Cardosa was emaciated and I hardly recognized him. The once vibrant couple was despondent. Although his abdominal computed tomography (CT) scan suggested new peritoneal metastases, the oncologist disagreed and pushed for more chemotherapy. I was concerned that his symptoms were related to progressive disease, but because of my now limited involvement with Mr. Cardosa's care, I felt that I was in no position to intervene.

I tried to convince myself that the oncologist should have kept me informed, but the truth was that I had let myself slide from participant to observer. Had I been more involved, the Cardosas, and perhaps, even the oncologist would have contacted me to take part in discussing the advisability of continued chemotherapy versus palliation and hospice care. My role in Mr. Cardosa's care would have been different, less central than previously, but it would still be important. The simple gesture of suggesting return visits would have allowed me to stay a functional part of the health care team and perhaps spared the couple some of the isolation and suffering they endured at the end.

Since my experiences with the Cardosas, I have begun to tell my patients newly diagnosed with serious illnesses that require subspecialty care (cancer, dialysis, and transplant) that I want to see them at intervals during their treatment and urge them to call me if they need advice. I have been struck by how positively they respond to this recommendation. I, too, feel better about my continued involvement with them.

My experience with the Cardosas brought into focus an important function of the General Internist. It made me strive to overcome concerns about status. Patients do suffer when the General Internist is not involved. We cannot afford to abandon our patients who develop serious illnesses even when subspecialists assume major responsibility for their treatment. If we do so, we deny our patients the best care; we deny ourselves the joys and sorrows of continuity; and we fail to fulfill our roles as General Internists. 